\title{
Introdución: la geografía económica en red
}

\author{
Maria del Pilar Alonso (a), Teresa Sá Marques (b), Helder Santos (c) \\ (a) Universitat de Lleida, p.alonso@geosoc.udl.cat \\ (b) CEGOT/Faculdade de Letras da Universidade do Porto, teresasamarques@gmail.com \\ (c) CEGOT/Faculdade de Letras da Universidade do Porto, hfcs75@hotmail.com
}

El imaginario de los territorios en red ha merecido una atención creciente por parte de la geografía en general y de la geografía económica en particular. La narrativa del territorio en red parte de análisis contenidos dentro de fronteras territoriales (redes locales, redes regionales), evolucionando hacia lecturas multi-escalares de relaciones que atraviesan esas fronteras territoriales, conectando, desconectando y volviendo a conectar lugares próximos o distantes (redes globales multi-escalares).

La exploración de los sistemas locales y regionales (de producción, de distribución, de información, de conocimiento, de innovación, de emprendimiento, etc.) surge, normalmente, integrada en sistemas más globales. Así el desarrollo económico de los territorios está asociado a su capacidad para establecer, anclar y aprovechar esas relaciones multi-escalares. Mientras tanto, las redes no son realidades abstractas. Son formas de interacción, implicando a individuos, ideas, organizaciones e instituciones, todos ellos insertados en territorios concretos, subrayando el carácter geográfico de las redes.

En este contexto, este libro cuenta con la participación de numerosos investigadores españoles y portugueses que abordan diferentes temas y responden a la diversidad de desafíos a los que ha de hacer frente la disciplina de Geografía Económica que cada vez se tiene que apoyar en la formación de redes para la comprensión de los procesos económicos. Esta diversidad reúne a los investigadores en torno a diferentes temáticas y escuelas, pero también en torno a proyectos de investigación colectiva que pretenden satisfacer tanto las actuales agendas de la investigación como las necesidades de la acción política.

Empezamos esta publicación con las aportaciones de Ricardo Méndez y José Luis Sánchez Hernández, en ambas nos encontramos con reflexione sobre los desafíos que se plantean en el futuro a la investigación en geografía económica.

Ricardo Méndez (2020) desarrolla su reflexión en torno a cuatro puntos interrelacionados: las transformaciones y demandas del entorno, los enfoques teóricos y metodológicos, el contexto institucional de la geografía económica y las temáticas emergentes para la geografía económica. José Luis Sánchez Hernández (2020) responde a este desafío abordando cuatro aspectos interrelacionados: transformaciones y demandas del entorno, contexto institucional de la disciplina, enfoques teóricos y metodológicos y temáticas de investigación emergentes.

El resto de libro hemos recogido aportaciones al funcionamiento territorial en red, centrados en cuatro ejes: Especialización inteligente; Tic, nuevos espacios económicos y ciudades inteligentes; Innovación económica y social; Comercio y servicios. Han sido varios los artículos centrados en el papel de la especialización inteligente que abordan el desarrollo intensivo del conocimiento, subrayado el papel de las instituciones -verbigracia, las instituciones de enseñanza superior - y reflejado los abordajes a diferentes escalas: europea, nacional, regional y local. Se identifican ecosistemas de innovación basados en redes de producción de conocimiento e innovación que involucran a diferentes actores y escalas territoriales. Estas redes representan conexiones asociadas a procesos de creación, producción y transferencia de conocimiento e innovación que pueden desencadenar una especialización compartida y una variedad relacionada entre los diferentes actores de la región.

Mário Vale et al. (2020) analizan la contribución de los institutos politécnicos a la concretización de las prioridades de la RIS3, mientras que Rui Gama et al. (2020) se centran en el papel de la Universidad de Coímbra en el desarrollo regional. Estas contribuciones permiten redactar orientaciones para una mejor coordinación entre las instituciones de enseñanza superior y la economía regional.

Helder Santos et al. (2020) abordan la especialización inteligente siguiendo las redes de proyectos de I+D+i H2020 ancladas en Portugal, atendiendo a la Estrategia Nacional de Investigación e Innovación para una Especialización Inteligente (ENEI) y las Estrategias Regionales de Investigación e Innovación para una Especialización Inteligente (RIS3). Pretenden caracterizar de este modo los ecosistemas de innovación (nacional y regionales) que parecen surgir en el periodo inicial de la implementación de la Estrategia H2020. 
Teresa Sá Marques et al. (2020) estudian el ecosistema propio de la Región Centro de Portugal y los subsistemas regionales de innovación a partir de los proyectos de innovación impulsados por organizaciones con sede en la región (entre 2007 y 2015). Identifican las redes organizativas y territoriales presentes en este ecosistema, y las tendencias de la especialización. Con ello, pretenden reconocer el conocimiento base que hace posible esos procesos, y los sectores de aplicación a los que van dirigidos, para caracterizar las capacidades regionales orientadas a la estrategia regional de especialización inteligente.

A través de las publicaciones internacionales relacionadas con el ébola (entre 1995 y 2017, en base a Web of Science), Thiago Mendes et al. (2020) identifican las regiones más destacadas en los procesos de producción de conocimiento a lo largo del tiempo, y confirman que las regiones de los países que ya sufrieron crisis epidemiológicas tienden a integrar en los periodos siguientes al grupo de regiones de referencia.

La política de especialización inteligente, tal como fue propuesta por la Unión Europea, se dirige a la escala regional. Sin embargo, a escala local existen instrumentos de política de desarrollo del territorio que son estructurales para el desarrollo y la planificación territorial. Teresa Sá Marques et al. (2020) defienden que los instrumentos de la política local deben ser valorados en los procesos de construcción y aplicación de las políticas de especialización inteligente, y pusieron a prueba el abordaje en la ejecución del plan director municipal de Oporto. Célia Ferreira et al. (2020) se centran todavía más territorialmente, e identificn y caracterizan los dominios de especialización inteligente más evidentes, las dinámicas recientes y el papel de la acción pública en la zona central de la ciudad de Oporto.

Ángel Miramontes Carballada (2020) reflexiona sobre la aeronáutica como sector estratégico a escala internacional que plantea grandes exigencias en materia de desarrollo tecnológico, por lo que constituye una apuesta de las políticas públicas. Se centra en la comunidad autónoma de Galicia, evidencia la red de innovación existente tanto a nivel de instituciones privadas como públicas, y plantea toda una serie de interrogantes.

Otros artículos giran en torno a las TIC, los nuevos espacios económicos y las ciudades inteligentes. Estas ciudades se centran en el crecimiento inteligente, en una economía de conocimiento y en la fuerza de los datos en red. Últimamente, las TIC han estado muy relacionadas con las ciudades gobernadas por dispositivos en red que generan una multitud de datos. A través del «internet de las cosas» circulan datos sobre flujos, transportes, calidad del aire, estacionamiento, consumo de energía o residuos urbanos, entre otros. Esos desarrollos movilizan a diferentes tipos de actores, desde empresas multinacionales de tecnología a empresas y comunidades locales, construyendo diferentes geografías locales y globales, unas más duraderas y otras claramente temporales.

Luís Carvalho (2020) combina nociones de geografía económica con estudios sociotécnicos de la innovación para explorar cómo una cartera «móvil» de tecnologías de redes/ciudades inteligentes (desarrollada por una empresa global de servicios públicos de energía) se ancla y recombina en diferentes ciudades y redes. R. González-Relaño et al. (2020) enuncian las variables clave que definen el desarrollo digital de hogares e individuos, e identifican siete tipos de regiones europeas con diferente grado de desarrollo digital. Rosa M. Jordá-Borrella et al. (2020) modelan los factores que influyen en la adopción de innovaciones de TIC por parte de las empresas. Este modelo permitie subrayar la importancia de los procesos de globalización de las empresas y la existencia de un ambiente tecnológico asociado a las TIC. Terminan señalando la importancia de que las instituciones públicas apoyen el incremento del conocimiento TIC, el fomento de la legislación relativa a las TIC y el establecimiento de criterios de contratación pública dirigidos a la innovación y adopción de TIC.

La innovación socioeconómica y resiliencia urbana como resultado del impacto de la crisis económica y financiera son cuestiones que obligan al incremento en la formación de redes desde diferentes perspectiva y que las aportaciones de estudios concretos sobre distintas ciudades permiten comprender. Julia Salom et al. (2020) conceptualizan la importancia de la innovación social en cuanto estrategia de desarrollo, y evaluan la red de actores y los niveles de colaboración en la ciudad de Valencia (España). Para finalizar, plantean un conjunto de recomendaciones de carácter político que ponen en evidencia la necesidad de incrementar la gobernanza transversal y participativa asociada al liderazgo público, para aumentar la resiliencia de los procesos de innovación social y ampliarlos desde el punto de vista territorial. A. López González (2020) aborda la resiliencia de dos ciudades medias españolas (León y Oviedo) a partir del impacto de la crisis económica y financiera. Identifica patrones comunes de resiliencia, pero también diferencias derivadas de las especificidades de cada ciudad. Los resultados permiten orientar la toma de decisiones en las redes de ciudadanos y establecer compromisos públicos.

Ángel Cebollada et al. (2020) dan fe del proceso de cambio social y económico en determinados barrios de las grandes ciudades. En Barcelona, los procesos de globalización y de financiarización de la ciudad se reflejan en la dinámica del mercado inmobiliario - altamente desregulado por el gran atractivo turístico- y en la acusada gentrificación urbana. Los procesos de internacionalización dan lugar a cambios socioeconómicos dirigidos a atraer segmentos residenciales de rentas altas. En este panorama, la bicicleta desempeña un papel simbólico, porque representa una mejor calidad de vida en la ciudad y mejora el atractivo residencial.

R. Vicente-Salar et al. (2020) analizan el papel de las economías externas en las evolución productiva del distrito textil de Trafalgar. El clúster textil ha desaparecido casi por completo para dar lugar a microclústeres creativos y de conocimiento retratados en un creciente número de coworkings en el espacio urbano de Barcelona. La popularización de esta ciudad como lugar innovador atrajo a empresas y recursos humanos altamente cualificados, lo que 
derivó en la aparición de un nuevo "soho barcelonés». A ello contribuyeron tanto la oferta de servicios de apoyo empresarial, formativos y de animación social como la dinamización de sentimientos de pertenencia en torno a ciertos barrios-comunidades intraurbanas.

El comercio y los servicios eran las actividades centrales por excelencia de las ciudades, ya que modelaban las estructuras urbanas y polarizaban su funcionamiento. Como consecuencia de los acusados procesos de urbanización experimentados sobre todo a finales del siglo XX, estas actividades se desplazaron a la periferia urbana, abandonando parcialmente el casco antiguo. En la actualidad, además de la actividad de las áreas urbanas centrales y los procesos de gentrificación urbana (turismo y nuevos residentes), el comercio y ciertos servicios se encuentran inmersos en un proceso de reestructuración espacial. Aunque que los mercadillos en la calle han desempeñado un importante papel en la dinamización y animación de las ciudades, hay también otro aspecto que hay que valorar en la escena de las ciudades como es la agricultura urbana ocupa una nueva centralidad en las estrategias urbanas De estos temas se ocupan los siguientes capítulos del libro.

Ana Vera et al. (2020) analizan ocho ciudades medias de Cataluña y, basándose en el comercio, se centran en los usos y las dinámicas de los espacios públicos de los centros urbanos tradicionales en cuanto lugares cruciales para la producción de identidades y la intensificación de sociabilidades urbanas.

Miguel Saraiva et al. (2020) relacionan el comercio con los patrones morfológicos y analizan las jerarquías intraurbanas de la ciudad de Oporto. Al introducir en el análisis variables relacionadas con la proximidad geográfica, la morfología y los propios establecimientos comerciales permiten mejorar el conocimiento de la estructura comercial y la gestión del comercio urbano con vistas a un acceso más equitativo.

Márcio Ferreira et al. (2020) se centran en la animación urbana a partir de los mercadillos de calle en Oporto y Barcelona durante la crisis económica y financiera posterior a 2008. Su papel durante la crisis y después de ella se vio alterado. Las actividades vinculadas a una economía alternativa emergieron como respuesta a un acusado aumento del desempleo durante la crisis. Sin embargo, tanto lo que ofrecen como la demanda que atraen reproducen la segmentación socioespacial de las propias ciudades. Tras la crisis, desempeñaron funciones de animación urbana alternativa, si bien todavía siguen reproduciendo la segmentación socioespacial anteriormente existente.

Maria del Pilar Alonso et al. (2020) abordan la reestructuración de las redes bancarias y sus repercusiones espaciales en tiempos de crisis en Lleida (España) y Presidente Prudente (Brasil), entre 2008 y 2016. En las últimas décadas se han registrado cambios globales en la organización del sistema financiero -mayores interdependencias globales que se han traducido en la concentración económica del sector y en un incremento de los servicios a través de internet- que conllevan una mayor selectividad en la distribución territorial de las sucursales y agencias bancarias, y generan nuevos patrones espaciales en las ciudades.

La agricultura urbana responde a la demanda diaria de los consumidores residentes en una ciudad o metrópolis a través del uso del suelo disponible en las zonas urbanas y periurbanas. I.M. Madaleno (2020) se centró en la agricultura urbana presente en la ciudad de Panamá -cultivo de alimentos, especias y plantas medicinales- y en el comercio de plantas americanas y exóticas y sus usos.

Las infraestructuras siguen influyendo en las dinámicas del desarrollo económico. Por otro lado, las trayectorias culturales o económicas pueden fomentar nuevas dinámicas de cooperación que enlazan a los territorios.

David Ramos-Pérez (2020) evidencian la generalización de los incentivos públicos para la creación de nuevas conexiones aéreas debido a la liberalización del mercado comunitario del transporte aéreo. A partir del Fondo de

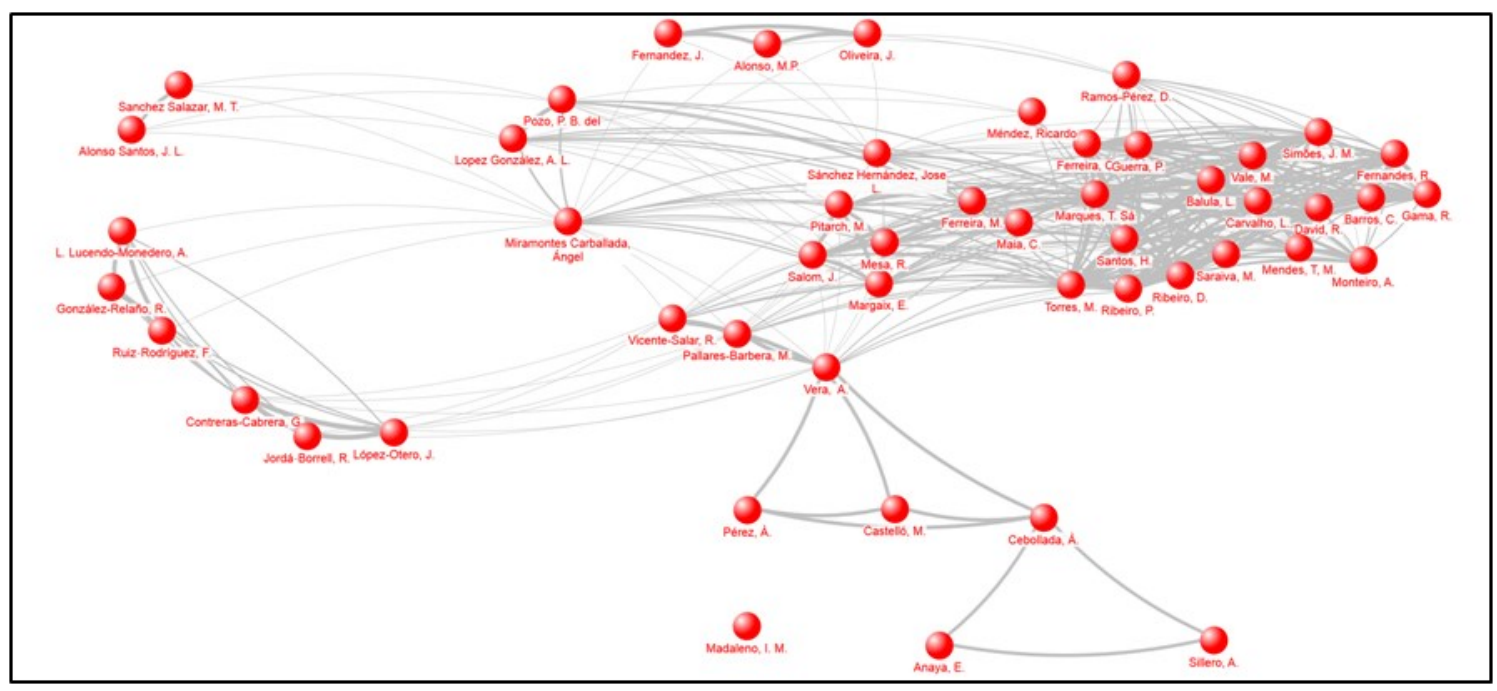

Figura 1. Proximidad de los autores de acuerdo la bibliografía común 
Desarrollo de Vuelos, en vigor desde 2014 en las islas Canarias - un destino turístico maduro y con una elevada conectividad aérea-, evalua el impacto de tales incentivos teniendo en cuenta los objetivos previamente definidos.

Las relaciones económicas entre España y México en la era de la globalización reflejan identidades históricas y afinidades culturales entre ambos países. José L. Alonso Santos et al. (2020) demuestran que la proximidad y la afinidad cultural deben ser incentivadas, ya que permiten abrir camino a nuevas inversiones económicas y al intercambio cultural. México puede convertirse en una plataforma de acceso al mercado centroamericano si se produce una apuesta estratégica por una mayor cooperación científica, cultural y económica.

En conclusión, La Geografía de las Redes Económicas y la Geografía Económica en Red permite incluir diferentes actividades de investigación llevadas a cabo por la comunidad científica del grupo de Geografía Económica de España, al que se sumó un importante número de investigadores de Portugal (figura 1). Al analizar los autores citados en la bibliografía de cada uno de los artículos de esta publicación, se pueden detectar varias comunidades científicas construidas a partir de esas referencias bibliográficas comunes. Los autores más citados en los artículos de esta publicación son R. Boschma, D. F. Campbell, E. G. Carayannis, P. A. Balland, H. Bathelt, R. Camagni, J. Glückler, B. A. Lundvall, R. Méndez y J. L. Sánchez Hernández.

\section{REFERENCIAS BIBLIOGRÁFICAS}

ALONSO, M.P; OLIVEIRA, J.; FERNÁNDEZ, F. (2020). Dinámicas espaciales recientes de las redes bancarias de España y Brasil: un estudio de las ciudades de Lleida y Presidente Prudente. In: M. Pilar Alonso Logroño, Teresa Sá Marques \& Helder Santos (Coord.), La Geografía de las Redes Económicas Y la Geografía Económica en Red, Porto, Faculdade de Letras da Universidade do Porto, Asociación de Geógrafos Españoles: 177-188. DOI: https://doi.org/10.21747/9789898969460/geoa17

ALONSO SANTOS, J. L.; SANCHEZ SALAZAR, M. T. (2020). Relaciones económicas España-México en la era de la globalización. Las exportaciones de España. In: M. Pilar Alonso Logroño, Teresa Sá Marques \& Helder Santos (Coord.), La Geografía de las Redes Económicas Y la Geografía Económica en Red, Porto, Faculdade de Letras da Universidade do Porto, Asociación de Geógrafos Españoles: 237-247. DOI: https://doi.org/10.21747/9789898969460/geoa23

CARVALHO, L. (2020). Smart city research metes the geography of innovation. The smart city programme of Enel in Rio de Janeiro and Santiago de Chile. In M. Pilar Alonso Logroño, Teresa Sá Marques \& Helder Santos (Coord.), La Geografía de las Redes Económicas Y la Geografía Económica en Red, Porto, Faculdade de Letras da Universidade do Porto, Asociación de Geógrafos Españoles: 91-98. DOI: https://doi.org/10.21747/9789898969460/geoa8

CEBOLLADA, À.; SILLERO, A.; ANAYA, E. (2020). Indagando en Sant Antoni (Barcelona) en torno a la relación de la bicicleta con la gentrificación. In: M. Pilar Alonso Logroño, Teresa Sá Marques \& Helder Santos (Coord.), La Geografía de las Redes Económicas Y la Geografía Económica en Red, Porto, Faculdade de Letras da Universidade do Porto, Asociación de Geógrafos Españoles: 189-197. DOI: https://doi.org/10.21747/9789898969460/geoa18

FERREIRA, C.; MARQUES, T. SÁ; GUERRA, P. (2020). A especialização inteligente na área central da cidade do Porto: domínios, ação pública e desafios. In M. Pilar Alonso Logroño, Teresa Sá Marques \& Helder Santos (Coord.), La Geografía de las Redes Económicas Y la Geografía Económica en Red, Porto, Faculdade de Letras da Universidade do Porto, Asociación de Geógrafos Españoles: 81-87. DOI: https://doi.org/10.21747/9789898969460/geoa7

FERREIRA, M.; MAIA, C.; MARQUES, T. SÁ (2020). As feiras de rua em contextos metropolitanos: Porto e Barcelona. In: M. Pilar Alonso Logroño, Teresa Sá Marques \& Helder Santos (Coord.), La Geografía de las Redes Económicas Y la Geografía Económica en Red, Porto, Faculdade de Letras da Universidade do Porto, Asociación de Geógrafos Españoles: 219-225. DOI: https:// doi.org/10.21747/9789898969460/geoa21

GAMA, R.; BARROS, C.; DAVID, R.; FERNANDES, R. (2020). Especialização inteligente e redes de conhecimento. A Universidade de Coimbra num contexto de globalização. In: M. Pilar Alonso Logroño, Teresa Sá Marques \& Helder Santos (Coord.), La Geografía de las Redes Económicas Y la Geografía Económica en Red, Porto, Faculdade de Letras da Universidade do Porto, Asociación de Geógrafos Españoles: 55-64. DOI: https://doi.org/10.21747/9789898969460/geoa5

LOPEZ GONZÁLEZ, A. L.; POZO, P. B. del (2020). Resiliencia en ciudades medias españolas: praxis económica y evidencias sociales en León y Oviedo. In: M. Pilar Alonso Logroño, Teresa Sá Marques \& Helder Santos (Coord.), La Geografía de las Redes Económicas Y la Geografía Económica en Red, Porto, Faculdade de Letras da Universidade do Porto, Asociación de Geógrafos Españoles: 167-175. DOI: https://doi.org/10.21747/9789898969460/geoa16

JORDÁ-BORRELLA, R. M.; LÓPEZ-OTERO, J.; CONTRERAS-CABRERA, G. (2020). Factores de mayor incidencia en la adopción de innovaciones tic a escala de pais. Importancia de las relaciones directas e indirectas entre factores. In: M. Pilar Alonso Logroño, Teresa Sá Marques \& Helder Santos (Coord.), La Geografía de las Redes Económicas Y la Geografía Económica en Red, Porto, Faculdade de Letras da Universidade do Porto, Asociación de Geógrafos Españoles: 109-117. DOI: https:// doi.org/10.21747/9789898969460/geoa10

GONZÁLEZ-RELAÑO, R.; L. LUCENDO-MONEDERO, A.; RUIZ-RODRÍGUEZ, F. (2020). El desarrollo digital de los hogares e individuos. una tipología de regiones europeas. In: M. Pilar Alonso Logroño, Teresa Sá Marques \& Helder Santos (Coord.), La Geografía de las Redes Económicas Y la Geografía Económica en Red, Porto, Faculdade de Letras da Universidade do Porto, Asociación de Geógrafos Españoles: 99-107. DOI: https://doi.org/10.21747/9789898969460/geoa9

MADALENO, I.M. (2020). Spices and Medicines Cultivated and Traded in Panama-City. In: M. Pilar Alonso Logroño, Teresa Sá 
Marques \& Helder Santos (Coord.), La Geografía de las Redes Económicas Y la Geografía Económica en Red, Porto, Faculdade de Letras da Universidade do Porto, Asociación de Geógrafos Españoles: 249-257. DOI: https://doi.org/10.21747/9789898969460/ geoa24

MARQUES, T. SÁ; SANTOS, H.; RIBEIRO, D.; RIBEIRO, P. (2020). Especialização Inteligente à Escala Urbana: Análise Dirigida ao Diagnóstico do PDM da Cidade do Porto. In: M. Pilar Alonso Logroño, Teresa Sá Marques \& Helder Santos (Coord.), La Geografía de las Redes Económicas Y la Geografía Económica en Red, Porto, Faculdade de Letras da Universidade do Porto, Asociación de Geógrafos Españoles: 65-79. DOI: https://doi.org/10.21747/978989896a9460/geoa6

MARQUES, T. SÁ; SANTOS, H.; RIBEIRO, P. (2020). Redes de inovação no ecossistema da Região Centro de Portugal. In: M. Pilar Alonso Logroño, Teresa Sá Marques \& Helder Santos (Coord.), La Geografía de las Redes Económicas Y la Geografía Económica en Red, Porto, Faculdade de Letras da Universidade do Porto, Asociación de Geógrafos Españoles: 141-150. DOI: https://doi.org/10.21747/9789898969460/geoa13

MENDES, T. M.; MARQUES, T. SÁ; MONTEIRO, A.; CARVALHO, L. (2020). Identificando Regiões de Referência na produção de conhecimento: o caso das publicações científicas voltadas para o Ébola. In: M. Pilar Alonso Logroño, Teresa Sá Marques \& Helder Santos (Coord.), La Geografía de las Redes Económicas Y la Geografía Económica en Red, Porto, Faculdade de Letras da Universidade do Porto, Asociación de Geógrafos Españoles: 151-158. DOI: https://doi.org/10.21747/9789898969460/ geoa14

MÉNDEZ, R. (2020). Desafíos de futuro para la investigación en Geografía Económica. In: M. Pilar Alonso Logroño, Teresa Sá Marques \& Helder Santos (Coord.), La Geografía de las Redes Económicas Y la Geografía Económica en Red, Porto, Faculdade de Letras da Universidade do Porto, Asociación de Geógrafos Españoles: 17-20. DOI: https://doi.org/10.21747/9789898969460/ geoa2

MIRAMONTES CARBALLADA, ÁNGEL (2020). La aeronáutica como sector estratégico en España. Situación actual y perspectivas en Galicia. In: M. Pilar Alonso Logroño, Teresa Sá Marques \& Helder Santos (Coord.), La Geografía de las Redes Económicas Y la Geografía Económica en Red, Porto, Faculdade de Letras da Universidade do Porto, Asociación de Geógrafos Españoles: 129138. DOI: https://doi.org/10.21747/9789898969460/geoa12

RAMOS-PÉREZ, D. (2020). El Fondo de Desarrollo de Vuelos de Canarias: incentivos públicos para nuevas rutas aéreas en un destino turístico maduro. In: M. Pilar Alonso Logroño, Teresa Sá Marques \& Helder Santos (Coord.), La Geografía de las Redes Económicas Y la Geografía Económica en Red, Porto, Faculdade de Letras da Universidade do Porto, Faculdade de Letras da Universidade do Porto, Asociación de Geógrafos Españoles: 227-235. DOI: https://doi.org/10.21747/9789898969460/ geoa22

SALOM, J.; PITARCH, M.; MESA, R.; MARGAIX, E. (2020). El papel de las redes en la innovación social: la ciudad de Valencia. In: M. Pilar Alonso Logroño, Teresa Sá Marques \& Helder Santos (Coord.), La Geografía de las Redes Económicas Y la Geografía Económica en Red, Porto, Faculdade de Letras da Universidade do Porto, Asociación de Geógrafos Españoles: 159-165. DOI: https://doi.org/10.21747/9789898969460/geoa15

SÁNCHEZ HERNÁNDEZ, JOSE L. (2020). Otras economías, otros espacios, otros retos para la Geografía Económica. In: M. Pilar Alonso Logroño, Teresa Sá Marques \& Helder Santos (Coord.), La Geografía de las Redes Económicas Y la Geografía Económica en Red, Porto, Faculdade de Letras da Universidade do Porto, Asociación de Geógrafos Españoles: 11-16. DOI: https:// doi.org/10.21747/9789898969460/geoa1

SANTOS, H.; MARQUES, T. SÁ; RIBEIRO, P.; TORRES, M. (2020). Especialização inteligente: as redes de projetos europeus H2020 com ancoragem em Portugal. In: M. Pilar Alonso Logroño, Teresa Sá Marques \& Helder Santos (Coord.), La Geografía de las Redes Económicas Y la Geografía Económica en Red, Porto, Faculdade de Letras da Universidade do Porto, Asociación de Geógrafos Españoles: 33-54. DOI: https://doi.org/10.21747/9789898969460/geoa4

SARAIVA, M.; MARQUES, T. SÁ; RIBEIRO, D.; RIBEIRO, P. (2020). Multi-diversity clusters of comercial activities in the city of Porto: how neighbourhood concentrations shape the municipal hierarchy. In: M. Pilar Alonso Logroño, Teresa Sá Marques \& Helder Santos (Coord.), La Geografía de las Redes Económicas Y la Geografía Económica en Red, Porto, Faculdade de Letras da Universidade do Porto, Asociación de Geógrafos Españoles: 209-217. DOI: https://doi.org/10.21747/9789898969460/ geoa20

VALE, M.; BALULA, L.; CARVALHO, L.; SIMÕES, J. M. (2020). Instituições de Ensino Superior e Especialização Inteligente: o contributo dos Institutos Superiores Politécnicos para a Implementação da RIS3 em Portugal. In: M. Pilar Alonso Logroño, Teresa Sá Marques \& Helder Santos (Coord.), La Geografía de las Redes Económicas Y la Geografía Económica en Red, Porto, Faculdade de Letras da Universidade do Porto, Asociación de Geógrafos Españoles: 23-31. DOI: https:// doi.org/10.21747/9789898969460/geoa3

VERA, A.; CEBOLLADA, À.; PÉREZ, A; CASTELLÓ, M. (2020). Propuesta metodológica para el estudio del uso y dinámicas del espacio público de las áreas comerciales de los centros urbanos. In: M. Pilar Alonso Logroño, Teresa Sá Marques \& Helder Santos (Coord.), La Geografía de las Redes Económicas Y la Geografía Económica en Red, Porto, Faculdade de Letras da Universidade do Porto, Asociación de Geógrafos Españoles: 201-207. DOI: https://doi.org/10.21747/9789898969460/geoa19

VICENTE-SALAR, R.; PALLARES-BARBERA M.; VERA-MARTIN, A. (2020). De clústeres a microclústeres: coworkings como nuevos espacios económicos en el Distrito Textil de Trafalgar en Barcelona. In: M. Pilar Alonso Logroño, Teresa Sá Marques \& Helder Santos (Coord.), La Geografía de las Redes Económicas Y la Geografía Económica en Red, Porto, Faculdade de Letras da Universidade do Porto, Asociación de Geógrafos Españoles: 119-128. DOl: https://doi.org/10.21747/9789898969460/geoa11 